\title{
Probing the redox activity of T-lymphocytes deposited at electrode surfaces with voltammetric methods
}

\author{
Ivan Bogeski ${ }^{1, *}$, Valentin Mirčeski ${ }^{2}$ and \\ Markus Hoth ${ }^{1}$ \\ ${ }^{1}$ Department of Physiology, Saarland University, \\ Homburg, Germany \\ ${ }^{2}$ Institute of Chemistry Faculty of Natural Sciences \\ and Mathematics, "Sv Kiril i Metodij" University, \\ Skopje, Republic of Macedonia
}

\begin{abstract}
Background: Reactive oxygen species and redox signaling play an important role in the regulation of many vital biological processes. However, they are also tightly connected with many pathological conditions. The detection and evaluation of these signaling events are very often accompanied with great difficulties. In this article, we describe the development of a novel electrochemically-based technique for monitoring the cellular redox state.

Methods and results: T-cells were attached on the surface of a working electrode, which was modified with 2-palmitoylhydroquinone as a redox mediator. Using cyclic voltammetry, we were able to indirectly (via the redox mediator) monitor an electron transport from the cells towards the working electrode, which enabled us to evaluate the redox activity of the cells. Conclusions: This new technique is rather simple and sensitive and may be used in the future as a valid diagnostic procedure in various branches of biomedical science.
\end{abstract}

Clin Chem Lab Med 2008;46:197-203.

Keywords: reactive oxygen species; redox signaling; T-cell; transmembrane electron-transfer; voltammetry.

\section{Introduction}

The transfer of free electrons $\left(\mathrm{e}^{-}\right)$across the plasma membrane and inside cells between various electron donors and acceptors is important for the regulation of many physiological processes (1-3). These chemical reactions, which occur as a result of electron $\left(\mathrm{e}^{-}\right)$ transfer, are usually referred to as redox reactions where the term "redox" is used for reduction (red) and oxidation (ox). Some chemical redox reactions at cellular membranes are followed by transfer of protons $\left(\mathrm{H}^{+}\right)$in the opposite direction. The chemical re-

*Corresponding author: Ivan Bogeski, Department of Physiology, Saarland University, Homburg, Germany Phone: + 49-6841-16-26452, Fax: +49-6841-16-26060 E-mail: ivan.bogeski@uks.eu

Received July 14, 2007; accepted September 6, 2007; previously published online December 13, 2007 actions, which involve $\mathrm{H}^{+}$transfer, are known as deprotonation and protonation. The elimination of microbes following phagocytosis and mitochondrial ATP production illustrates the importance of these bio-electrochemical reactions $(1,2)$.

A wide group of enzymes and proteins regulate the general redox state of cells (2). One of the best-studied enzymes of this group is the NADPH-oxidase, which is located in the plasma membrane (4). When activated, it transports electrons from cytosolic NADPH ( $\mathrm{e}^{-}$donor) to molecular oxygen ( $\mathrm{e}^{-}$acceptor) in the extracellular space, thus facilitating generation of reactive oxygen species (ROS) (5). As mentioned above, this $\mathrm{e}^{-}$transfer is followed by $\mathrm{H}^{+}$transfer in the opposite direction. The mechanism of this $\mathrm{H}^{+}$ transfer is not fully understood. There is evidence that NADPH-oxidase can transfer protons by itself. However, there are other studies that implicate a completely independent pathway via proton channels (6). Nevertheless, it is well known that phagocytes and T-cells express this enzyme (7) and that it plays a major role in the regulation of the redox state of these cells. The $\mathrm{e}^{-}$transfer facilitated by the NADPH-oxidase leads to a generation of $\mathrm{O}_{2}^{-}$, a common $\operatorname{ROS}(4,7,8)$. ROS are important for the regulation of the immune response (2), which is evident from the antimicrobial effect of ROS generated by the phagocytes (oxidative burst) during microbial invasion (8).

Relatively small amounts of ROS, in comparison to the amount generated during phagocytosis, are also generated by T-cells and they have a regulatory role in many signaling cascades. The origin of these free radicals is mainly the T-cell NADPH-oxidase (7), but also mitochondria $(9,10)$, xantine oxidase and the arachidonic acid pathway (11). It has been shown that ROS regulate the activity of many proteins, including phosphatases and kinases, ion channels, transcription factors, etc. (2). Interestingly, many cancer cells have an increased redox activity $(12,13)$. Hence, detection of the redox state of any given cell population may be a valuable diagnostic procedure. A measurement of electron and proton currents across the plasma membrane is one method to determine the redox state of the cells. Until now, this task has been achieved only by using the patch-clamp methodology and was accompanied by some problems, such as the fast depletion of the NADPH-oxidase cytosolic-substrate NADPH. These problems are even more pronounced during whole-cell measurements used to record transmembrane electron currents (6).

Electrochemistry is a powerful tool to study redox potentials and activities of many compounds. Electrochemical techniques are particularly useful in analyzing redox activities of membrane proteins, including ion transporters and channels. In the current study, 
we have developed a simple method for probing the redox activity of T-cells. The method utilizes conventional cyclic voltammetry in combination with a specially designed electrode on the surface of which the cells are spontaneously immobilized. Before attachment of the cells, the surface of the electrode was modified with a redox mediator that facilitates the electron transfer between the electrode and the immobilized cells. The overall electrochemical response is based on an electrochemical regenerative catalytic mechanism (14).

\section{Materials and methods}

\section{Chemicals and buffers}

The redox mediator 2-palmitoylhydroquinone $\left(\mathrm{H}_{2} \mathrm{O}\right) \quad(99 \%$ purity) was purchased from Merck (Merck KGaA, Darmstadt, Germany) and decamethylferrocene (DMFC) was from Fluka (Sigma-Aldrich Chemie $\mathrm{GmbH}$, Munich, Germany). Both redox mediators were used as received without further purification. The redox mediator polyphenotiazine (PPTA), a redox polymer, was synthesized as described previously (15). All redox mediators were dissolved in 1,2-dichloroethane (DCE) at a concentration of $10 \mathrm{mM}$. Fura 2/AM was purchased from Molecular Probes (Invitrogen $\mathrm{GmbH}$, Karlsruhe, Germany) and all other chemicals were from Sigma (highest grade) (Sigma-Aldrich Chemie $\mathrm{GmbH}$, Munich, Germany). All measurements were performed in Ringer's solution, which consisted of (in $\mathrm{mM}$ ): $155 \mathrm{NaCl}, 4.5 \mathrm{KCl}, 1$ EGTA, $2 \mathrm{MgCl}_{2}, 10$ D-glucose and 5 HEPES $(\mathrm{pH} 7.4$ with $\mathrm{NaOH}$.

\section{Cell culture}

E6-1 Jurkat T-cells (ATCC, LGC Promochem GmbH, Wesel, Germany) were grown in RPMI 1640 culture medium supplemented with $10 \%$ fetal calf serum and penicillin-streptomycin, at $37^{\circ} \mathrm{C}$ in a humidified atmosphere $\left(5 \% \mathrm{CO}_{2}\right)$.

\section{Cell imaging}

Cells were visualized by light and fluorescence microscopy using an Olympus IX 70 microscope (Olympus Deutschland $\mathrm{GmbH}$, Hamburg, Germany) equipped with a $20 \times$ (UApo/ 340, N.A. 0.75) objective, with a Polychrome V Monochromator (TILL Photonics GmbH, Graefelfing, Germany) and a charge-coupled device camera TILL Imago (TILL Photonics $\mathrm{GmbH}$, Graefelfing, Germany). To visualize cells on the electrode, they were loaded with $5 \mu \mathrm{M}$ Fura 2/AM at room temperature for $30 \mathrm{~min}$. The images were analyzed using TILL Vision software (TILL Photonics $\mathrm{GmbH}$, Graefelfing, Germany) as described previously (16).

\section{Electrochemistry}

Cyclic voltammetry (CV) measurements were carried out with a conventional 3-electrode potentiostat ( $\mu$-Autolab Model III, Eco-Chemie, Utrecht, Netherlands). An Ag/AgCl $(3 \mathrm{M} \mathrm{KCl})$ was used as a reference electrode, while a Pt wire was the counter electrode. The working electrode was a disk electrode of paraffin-impregnated graphite (PIGE), edge plane pyrolytic graphite (EPPGE) and gold (Au). The procedure for preparing PIGE has been described previously (17).

Before modification of PIGE with the redox mediators and T-cells, the electrode was abraded with a SiC paper (600), rinsed with pure water, dried on air, and finally polished to a smooth surface on a white paper. EPPGE was abraded with a SiC paper (600), sonicated for $30 \mathrm{~s}$, rinsed with pure water, acetone and dried on air. The Au electrode was abraded with $\mathrm{Al}_{2} \mathrm{O}^{3}$ slurry and rinsed with pure water.

The electrode surface was modified by imposing a $1 \mu \mathrm{L}$ of DCE solution (with the aid of a micropipette) containing one of the redox mediators. After evaporation of the solvent, the electrode was immersed into a buffer solution to record repetitive cyclic voltammograms to examine the redox activity and stability of the response. After reaching a stable steady-state voltammetric response, the electrode was dried on air and a $1 \mu \mathrm{L}$ suspension of T-cells was applied to the electrode surface with the aid of a micropipette to allow cell adsorption via membrane proteins $(18,19)$. After a few minutes, the electrode with adsorbed T-cells was again immersed in the same buffer solution and voltammograms were recorded in a conventional manner. All experiments were performed at room temperature.

\section{Results}

The electrode assembly used to probe the redox activity of Jurkat T-cells is presented in Figure 1A. It consists of a specially designed PIGE modified with the redox active compound $\mathrm{H}_{2} \mathrm{Q}$. The redox probe possesses a long hydrophobic palmitoyl substituent, whereas the hydroquinone ring is hydrophilic and redox active (see the inset of Figure 1A). Considering the amphiphilic properties of $\mathrm{H}_{2} \mathrm{Q}$, it is plausible to assume that the molecule is adsorbed on the electrode surface through the semi-linear hydrophobic palmitoyl tail, whereas the hydrophilic hydroquinone ring is oriented toward the aqueous phase. Such orientation was also observed in the case of adsorbed ubiquinone-10, which has a similar molecular structure to $\mathrm{H}_{2} \mathrm{O}(20)$. A detailed study of the redox behavior of $\mathrm{H}_{2} \mathrm{Q}$ is given in ref. (21). The hydroquinone ring can undergo an overall redox transformation through a $2 \mathrm{e} / 2 \mathrm{H}^{+}$chemically reversible process to the quinone form (Q). It is generally accepted that the redox transformation of the hydroquinone/quinone redox couple proceeds as a two-step mechanism via formation of a short-life semiquinone $\mathrm{HQ}$ radical (20). In most of the cases, expel of the first electron from the hydroquinone form is energetically more difficult than the second one, thus the overall process appears as a simultaneous two-electron exchange redox reaction. Typical cyclic voltammograms recorded in a physiological buffer at $\mathrm{pH} 7.4$ are depicted in Figure 1B. The voltammograms are rather stable over time and show a single peak pair corresponding to the redox couple $\mathrm{H}_{2} \mathrm{Q} / \mathrm{Q}$. The oxidation peak positioned in the positive current range reflects the oxidation of $\mathrm{H}_{2} \mathrm{Q}$ to $\mathrm{Q}$, whereas the negative reduction peak is due to the reverse reduction process of $\mathrm{Q}$ form that is electrochemically formed during the previous potential scan. The overall redox reaction is represented by the scheme:

$\mathrm{H}_{2} \mathrm{Q} \leftrightarrow \mathrm{Q}+2 \mathrm{H}^{+}+2 \mathrm{e}^{-}$

The large potential separation between the anodic and cathodic peaks implies a quasireversible electro- 


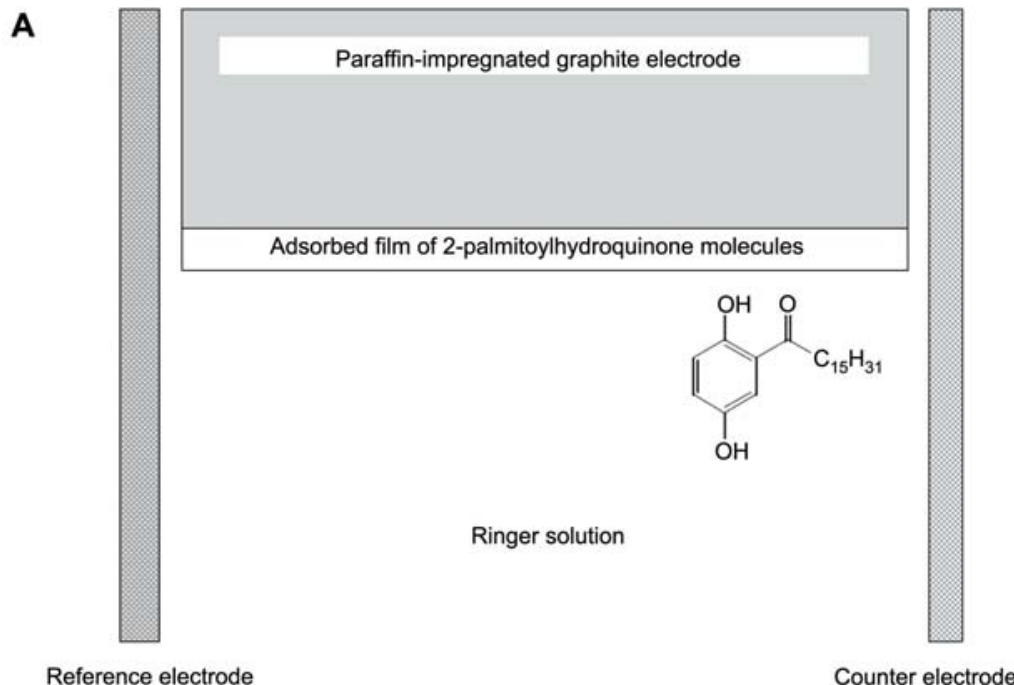

B

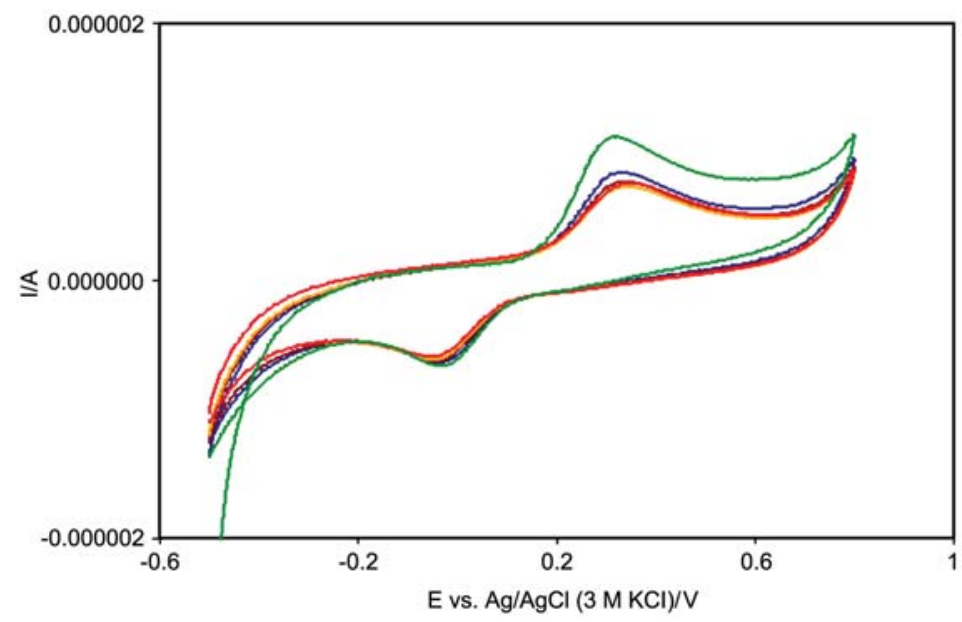

Figure 1 (A) Schematic representation of the electrochemical setup. The inset shows the molecular structure of the redox mediator 2-palmitoylhydroquinone. (B) Five consecutive cyclic voltammograms of the redox mediator $\mathrm{H}_{2} \mathrm{O}$ recorded at PIGE in Ringer's solution at $\mathrm{pH} 7.4$ and scan rate of $\mathrm{v}=50 \mathrm{mV} / \mathrm{s}$ are overlaid.

chemical process as a consequence of relatively slow kinetics of the electrode reaction [1]. To measure the redox activity of cells, we attached Jurkat T-cells onto the electrode as schematically shown in Figure 2A (see Materials and methods for details). To confirm the viability and the attachment of Jurkat T-cells on the PIGE surface, imaging experiments were conducted. Figure 2B shows fluorescent images of Jurkat T-cells loaded with fluorescent dye Fura 2/AM, attached on PIGE. In this case, the electrode was modified with $\mathrm{H}_{2} \mathrm{O}$ as a redox mediator. Fura 2 is a $\mathrm{Ca}^{2+}$ sensitive dye, and dying cells are not able to retain it in the cytosol. Most of the cells had normal Fura 2 signals, as usually observed in typical imaging experiments $(22,23)$.

In the presence of the T-cells, the voltammetric response of the electrode is significantly changed, exhibiting an increase of both oxidation and reduction peak amplitudes (Figure 3A). In particular, the enhancement of the oxidation peak current is much more pronounced than the reduction one. Though the oxidative peak current is shifted toward more positive potentials in the presence of T-cells, the oxidation current starts developing at a slightly less positive potential. In addition, as shown in Figure $3 \mathrm{~A}$, both oxidative and reductive peak currents increase in proportion to the amount of cells adsorbed on the electrode surface. In the absence of the redox probe, the immobilized cells were not capable of communicating directly with the electrode material. A control experiment conducted without redox probe but with cells is depicted in Figure 3B, showing no significant faradic processes, because there is no direct electron exchange between the electrode and the cells. Measuring the oxidative current at a particular potential of the cyclic voltammetric experiment, e.g., $0.41 \mathrm{~V}$, it has been established that the current in the presence of the redox probe and cells $(2.07 \mu \mathrm{A}$ and $2.83 \mu \mathrm{A}$ after the first and second addition of the cells, respectively) is higher than the sum of currents measured in separate experiments conducted only with cells $(0.55 \mu \mathrm{A})$ and the redox probe $\mathrm{H}_{2} \mathrm{O}(1.34 \mu \mathrm{A})$. All these results show that in the presence of the redox probe and the adsorbed T-cells, a catalytic electrode mechanism is 

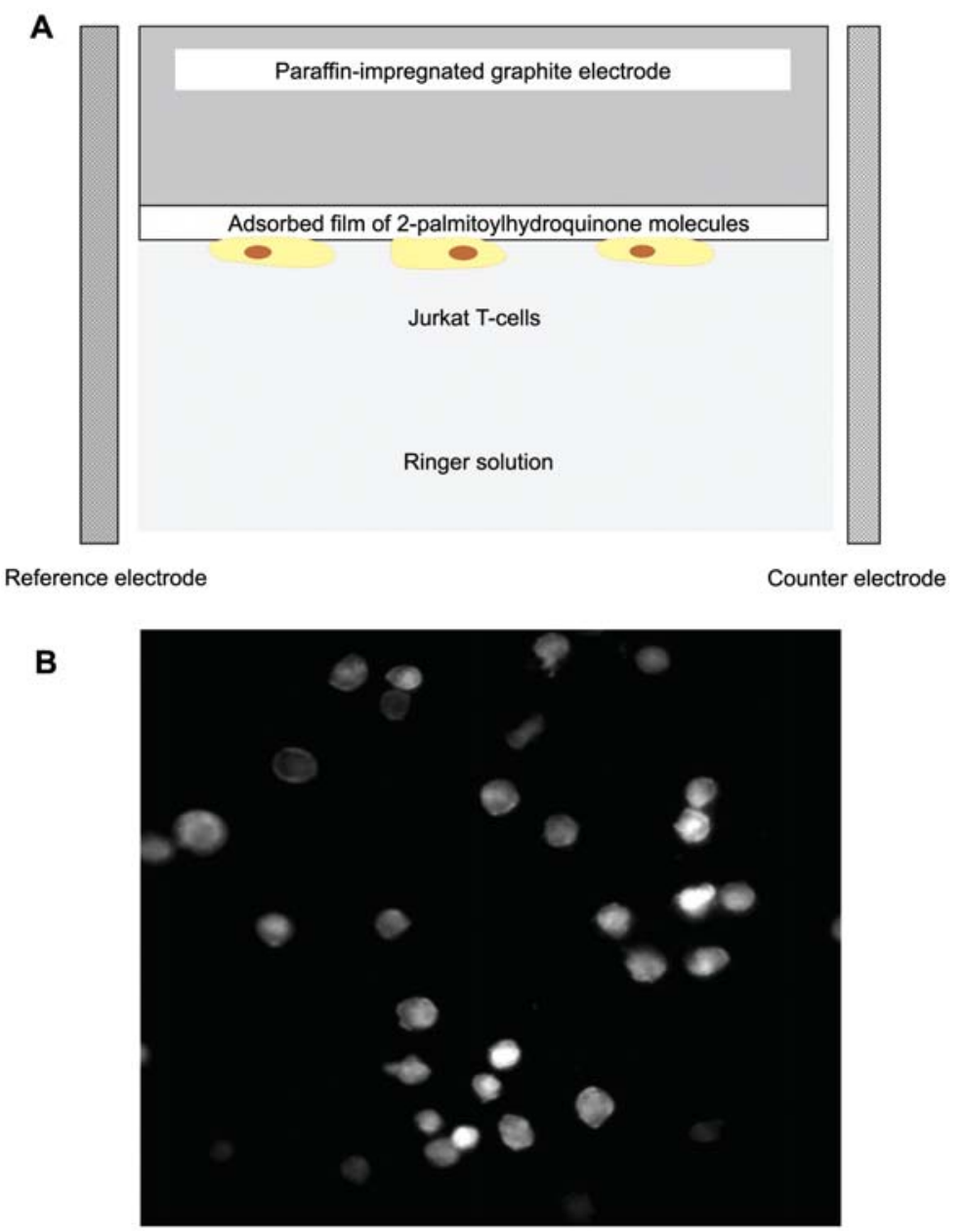

Figure 2 (A) Schematic representation of the electrochemical setup, modified with the redox mediator and Jurkat T-cells. (B) Fluorescent images of Fura 2/AM-loaded Jurkat T-cells attached to the PIGE electrode. The working electrode was modified with $\mathrm{H}_{2} \mathrm{O}$ as a redox mediator.

operative, where the adsorbed redox couple $\mathrm{H}_{2} \mathrm{Q} / \mathrm{O}$ acts as an electrochemical catalyst, facilitating the electron exchange between adsorbed cells and the electrode (24).

Considering the redox mechanism of the couple $\mathrm{H}_{2} \mathrm{Q} / \mathrm{Q}$ and the fact that the standard redox potential of the couple $\mathrm{HO} / \mathrm{Q}$ is more negative than $\mathrm{H}_{2} \mathrm{Q} / \mathrm{HQ}$. $(20,21)$, it can be concluded that the semiquinone radical $\mathrm{HQ}^{\circ}$ is the stronger oxidizing agent than the quinone form $\mathrm{Q}$. This implies the actual redox catalyst that shuttles electrons from the cells to the electrode is the semiquinone radical $\mathrm{HO}$, which is formed in situ on the electrode surface in the course of cyclic voltammetric experiment. Therefore, in an anodic direction of the potential sweep, the final result is the detection of a flux of electrons from the T-cells toward the electrode, mediated by the semiquinone radical, which is an intermediate of the electrochemical oxidation of the redox probe.

The redox reaction was further characterized by varying the scan rate of the application of the potential sweep in the voltammetric experiment. It is important to point out that the increase of the sweep rate in turn decreases the critical time of the voltammetric experiment, thus decreasing the time for interaction between the adsorbed cells and the semiquinone radical formed electrochemically on the electrode surface. For this reason, the ratio between the anodic-to-cathodic peak current decreased with the scan rate (Figure $3 \mathrm{C}$ ), which is typical for a catalytic electrode mechanism in cyclic voltammetry (14).

Considering the overall electrochemical phenomena observed in the presence of adsorbed T-cells, it is particularly intriguing that an electrocatalytic effect was also observed in the cathodic direction, related to the reduction of quinone form. To explain this effect, one has to consider that besides the detection of electron flux from the adsorbed cells to the electrode surface, adsorbed cells can also transfer electrons to the dissolved oxygen in the solution to form ROS, in agreement with the physiological function of the NADPH-oxidase (6). Therefore, in close proximity to the electrode surface, formation of various ROS is very likely. On the other hand, it is well known that adsorbed quinones are excellent electrochemical catalysts for the reduction of oxygen-related species (25). For these reasons, the cathodic peak current increases proportionally to the amount of T-cells adsorbed on 


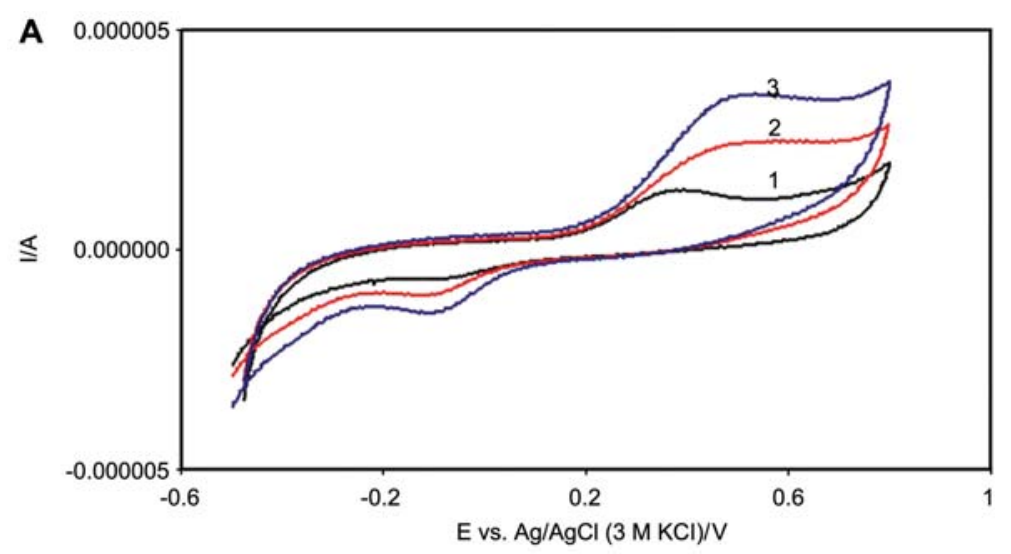

B
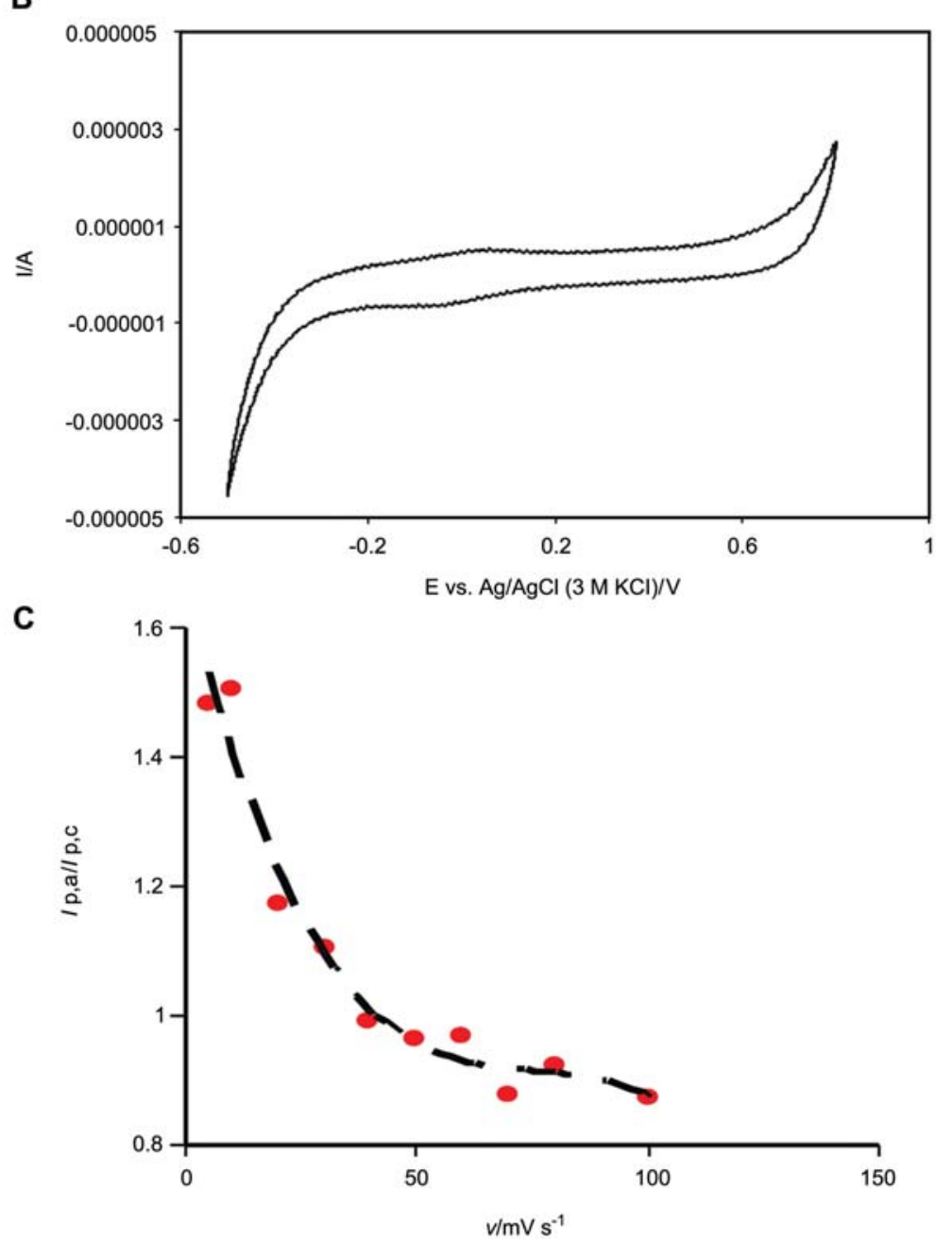

Figure 3 (A) Effect of Jurkat T-cells on the cyclic voltammetric response of $\mathrm{H}_{2} \mathrm{Q}$. (1) In the absence of Jurkat T-cells, (2) first addition of Jurkat T-cells, and (3) second addition of Jurkat T-cells. All other conditions are the same as for Figure 1B. (B) Effect of Jurkat T-cells on the cyclic voltammetric response recorded in the absence of the redox mediator. All conditions are the same as for Figure 1B. (C) Dependence of the anodic-to-cathodic peak current on the scan rate.

the electrode surface, reflecting the catalytic reduction of ROS. Again, the most probable electrocatalyst is the semiquinone radical $\mathrm{HQ}$ :

In addition to $\mathrm{H}_{2} \mathrm{Q}$, DMFC and PPTA were tested as redox mediators. DMFC can be reversibly oxidized to a stable cation $\mathrm{DMFC}^{+}$in a fast electrochemical reaction (26). However, in the presence of T-cells, the response of the electrode decreased (data not shown), implying that the DMFC/DMFC ${ }^{+}$redox couple cannot exchange electron with cells. Note that the decrease of the response could also be due to instability of the immobilization of the redox probe on the electrode surface in the course of the potential cycling. Contrary to DMFC, PPTA exhibited a similar effect to $\mathrm{H}_{2} \mathrm{Q}$, as shown in Figure 4. PPTA is a strongly hydrophobic redox polymer with no detectable solu- 


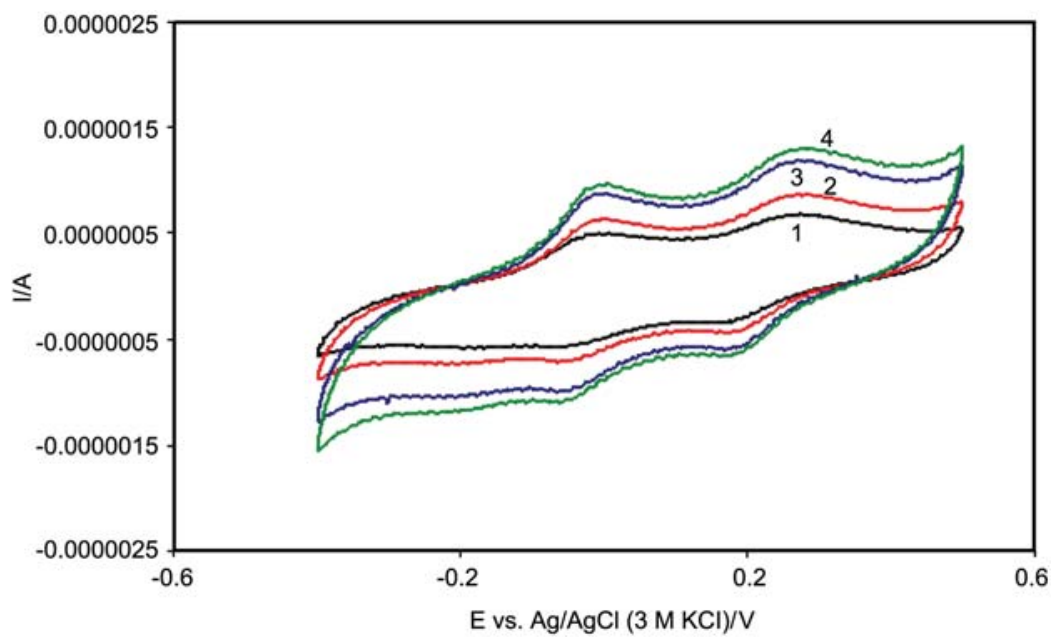

Figure 4 Effect of Jurkat T-cells on the cyclic voltammetric response of PPTA recorded with the PIGE electrode in Ringer's solution at $\mathrm{pH}$ 7.4. (1) In the absence of Jurkat T-cells, (2) first addition of Jurkat T-cells, (3) second addition of Jurkat T-cells, and (4) third addition of Jurkat T-cells. All experimental conditions are the same as for Figure 1B.

bility in water. PPTA can be oxidized in two independent chemically reversible steps on the electrode surface (Figure 4). Both redox steps are sensitive to the attached T-cells, though the catalytic effect is not as strong as in the case of $\mathrm{H}_{2} \mathrm{O}$. However, using PPTA as a redox probe, the background current also increases by adding T-cells (Figure 4) as a consequence of the capacitive current enhancement. This indicates the morphology of the electrode surface covered by the redox active polymer is significantly altered in the presence of T-cells. This unfavorable phenomenon is much less pronounced when using $\mathrm{H}_{2} \mathrm{O}$ as a redox mediator (Figure $3 \mathrm{~A}$ ). While the strong hydrophobicity of PPTA is in favor of its irreversible adsorption on the electrode surface, its strong hydrophobicity, on the other hand, inhibits the effective interaction with the cell membranes, which is needed to capture the electron flux from the immobilized cells.

The foregoing results imply that the amphiphilic properties of the redox probe are critical for the effective interactions with both the electrode and the immobilized cells. Of the three redox probes tested, $\mathrm{H}_{2} \mathrm{O}$ possesses well-balanced amphiphilic properties. The long palmitoyl tail enables strong and irreversible adsorption on the electrode surface. On the other hand, the hydrophilic hydroquinone moiety, together with its very specific redox chemistry involving formation of highly active redox intermediate, enables effective interactions with redox centers embedded in the cell membranes.

Beside the redox mediator, the overall methodology depends critically on the electrode material, which controls the efficiency of cell attachment. In addition to PIGE, experiments using EPPGE and Au electrodes were performed with $\mathrm{H}_{2} \mathrm{Q}$ as a redox mediator. The attachment of Jurkat T-cells to EPPGE and Au electrodes resulted in a decrease of the voltammetric response of the redox mediator (data not shown). The strong adsorption of the cells on these electrode materials might cause replacement and desorption of the redox mediator, precluding detection of the catalytic effect. Reproducible catalytic effects could only be reliably measured with PIGE. The microscopic structure of PIGE comprises clusters of graphite grains, interconnected by paraffin (17). The olefin structure of the paraffin allows strong interactions with the palmitoyl chain of $\mathrm{H}_{2} \mathrm{Q}$, whereas the graphite clusters remain free for cell immobilization. Moreover, the redox-active hydroquinone ring of the $\mathrm{H}_{2} \mathrm{O}$ molecule, which is not engaged in the adsorptive interactions with the electrode material, remains free to accommodate a position, which allows electron exchange with the cell membranes. Therefore, PIGE is a well-suited electrode material that enables noncompetitive and effective immobilization of both the redox mediator and T-cells, bringing them into intimate contact for mutual electron exchange.

\section{Discussion}

ROS and redox chemistry in general are very important for the regulation of many vital signaling events. The function of many proteins and lipids can be regulated by oxidation and reduction (2), and DNA is also susceptible to functional changes induced by ROS. In addition, redox signaling is also tightly connected with many pathological conditions. Free radicals are involved in pathogenesis of ageing, cancer, rheumatoid arthritis, cardiovascular disease, arteriosclerosis, hypertension, diabetes mellitus, and Parkinson's and Alzheimer's disease (27-31). The role of ROS as pathogens is also very well established.

Contrary to its great importance, the diagnostics, evaluation, and detection of redox signaling events in biological systems, in basic research as well as in clinical practice, is still a particularly difficult task and development of new and more sensitive methodologies is needed (32-35).

We introduce a novel electrochemically-based technique for the detection of electron transport across 
the cellular plasma membrane. Electron transfer is a valuable determinant of the whole redox state and redox potential of a living cell. Although we presume that at least part of the catalytic effect, which Jurkat T-cells have on the redox mediator, is facilitated by the NADPH-oxidase, electron transfer via some redoxactive ion channels, transporters or exchangers expressed in the plasma membrane may well contribute.

The new technique that we propose is rapid, sensitive, simple, and with some minor modifications could be applied as a valuable diagnostic procedure in different branches of bio-medical research.

\section{Acknowledgements}

This work was supported by the Deutsche Forschungsgemeinschaft (SFB 530, project A3) and Graduate College 845 "Molecular, physiological and pharmacological analysis of cellular membrane transport" (to M.H.) and a competitive grant from the Medical Faculty of the Saarland University (HOMFOR, to I.B.). V.M. also gratefully acknowledges the financial support of A. v. Humboldt-Stiftung.

\section{References}

1. Forman HJ, Torres M, Fukuto J. Redox signaling. Mol Cell Biochem 2002;234-235:49-62.

2. Droge W. Free radicals in the physiological control of cell function. Physiol Rev 2002;82:47-95.

3. Fridovich I. Fundamental aspects of reactive oxygen species, or what's the matter with oxygen? Ann NY Acad Sci 1999;893:13-8.

4. Lambeth JD. NOX enzymes and the biology of reactive oxygen. Nat Rev Immunol 2004;4:181-9.

5. Schrenzel J, Serrander L, Banfi B, Nusse O, Fouyouzi R, Lew DP, et al. Electron currents generated by the human phagocyte NADPH oxidase. Nature 1998;392:734-7.

6. Demaurex N, Petheo GL. Electron and proton transport by NADPH oxidases. Phil Trans R Soc Lond B Biol Sci 2005;360:2315-25.

7. Jackson SH, Devadas S, Kwon J, Pinto LA, Williams MS. T cells express a phagocyte-type NADPH oxidase that is activated after $T$ cell receptor stimulation. Nat Immunol 2004;5:818-27.

8. Forman HJ, Torres M. Signaling by the respiratory burst in macrophages. IUBMB Life 2001;51:365-71.

9. Brookes PS, Yoon Y, Robotham JL, Anders MW, Sheu SS. Calcium, ATP, and ROS: a mitochondrial love-hate triangle. Am J Physiol Cell Physiol 2004;287:C817-33.

10. Bogeski I, Bozem M, Sternfeld L, Hofer HW, Schulz I. Inhibition of protein tyrosine phosphatase $1 \mathrm{~B}$ by reactive oxygen species leads to maintenance of $\mathrm{Ca}^{2+}$ influx following store depletion in HEK 293 cells. Cell Calcium 2006;40:1-10.

11. Baud L, Ardaillou R. Reactive oxygen species: production and role in the kidney. Am J Physiol Renal Physiol 1986; 251:F765-76.

12. Fry FH, Jacob C. Sensor/effector drug design with potential relevance to cancer. Curr Pharm Design 12:4479-99.

13. Gius D, Spitz DR. Redox signaling in cancer biology. Antioxid Redox Signal 2006;8:1249-52.

14. Bard AJ, Faulkner, L.R. Electrochemical methods, fundamentals and applications. New York: John Wiley \& Sons, 2001.
15. Karyakin AA, Vagin MY, Ozkan SZ, Karpachova GP. Thermodynamics of ion transfer across the liquid/liquid interface at a solid electrode shielded with a thin layer of organic solvent. J Phys Chem B 2004;108:11591-5.

16. Quintana A, Schwarz EC, Schwindling C, Lipp P, Kaestner L, Hoth M. Sustained activity of calcium releaseactivated calcium channels requires translocation of mitochondria to the plasma membrane. J Biol Chem 2006;281:40302-9.

17. Scholz F, Meyer, B. Electroanalytical chemistry, a series of advances. New York: Marcel Dekker, 1998.

18. Rozhok S, Holz R. Electrochemical attachment of motile bacterial cells to gold. Talanta 2005;67:538-42.

19. Collier JH, Mrksich M. Engineering a biospecific communication pathway between cells and electrodes. Proc Natl Acad Sci USA 2006;103:2021-5.

20. Gordillo GJ, Schiffrin DJ. The electrochemistry of ubiquinone-10 in a phospholipid model membrane. Faraday Discuss 2000;116:89-107.

21. Mirceski V, Gulaboski R, Bogeski I, Hoth M. Redox chemistry of Ca-transporter 2-palmitoylhydroquinone in an artificial thin organic film membrane. J Phys Chem C 2007;111:6068-76.

22. Quintana A, Hoth M. Apparent cytosolic calcium gradients in T-lymphocytes due to fura-2 accumulation in mitochondria. Cell Calcium 2004;36:99-109.

23. Schwarz A, Tutsch E, Ludwig B, Schwarz EC, Stallmach

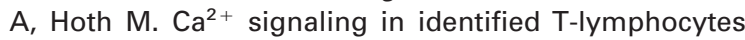
from human intestinal mucosa. Relation to hyporeactivity, proliferation, and inflammatory bowel disease. J Biol Chem 2004;279:5641-7.

24. Lovric M, Branica M. The simulation of the homogeneous catalytic reaction at a monolayer-film covered rotating-disk electrode. Electrochim Acta 1983;28:1261-7.

25. Sljukic B, Banks CE, Compton RG. An overview of the electrochemical reduction of oxygen at carbon-based modified electrodes. J Iranian Chem Soc 2005;2:1-25.

26. Mirkin MV, Richards TC, Bard AJ. Scanning electrochemical microscopy. 20. Steady-state measurements of the fast heterogeneous kinetics in the ferrocene/acetonitrile system. J Phys Chem 1993;97:7672-7.

27. Fruehauf JP, Meyskens FL Jr. Reactive oxygen species: a breath of life or death? Clin Cancer Res 2007;13:78994.

28. Kregel KC, Zhang HJ. An integrated view of oxidative stress in aging: basic mechanisms, functional effects, and pathological considerations. Am J Physiol Regul Integr Comp Physiol 2007;292:R18-36.

29. Paravicini TM, Touyz RM. Redox signaling in hypertension. Cardiovasc Res 2006;71:247-58.

30. Abramov AY, Duchen MR. The role of an astrocytic NADPH oxidase in the neurotoxicity of amyloid beta peptides. Phil Trans R Soc Lond B Biol Sci 2005;360: 2309-14.

31. Droge W. Oxidative stress and ageing: is ageing a cysteine deficiency syndrome? Phil Trans R Soc Lond B Biol Sci 2005;360:2355-72.

32. Cherubini A, Ruggiero C, Polidori MC, Mecocci P. Potential markers of oxidative stress in stroke. Free Radic Biol Med 2005;39:841-52.

33. Voss $P$, Siems W. Clinical oxidation parameters of aging. Free Radic Res 2006;40:1339-49.

34. Simic MG. DNA markers of oxidative processes in vivo: relevance to carcinogenesis and anticarcinogenesis. Cancer Res 1994;54(Suppl 7):1918-23s.

35. Tarpey MM, Fridovich I. Methods of detection of vascular reactive species: nitric oxide, superoxide, hydrogen peroxide, and peroxynitrite. Circ Res 2001;89:224-36. 